\title{
Open circuit voltage recovery in type II GaSb/GaAs quantum ring solar cells under high concentration
}

\author{
H. Fujita ${ }^{\text {a d }}$, *, P. J. Carrington ${ }^{\text {a }}$, M. C. Wagener ${ }^{\text {b }}$, J. R. Botha ${ }^{\text {b }}$, A. R. J. Marshall ${ }^{\text {a }}$, J. James ${ }^{\text {a }}$, \\ A. Krier ${ }^{a}$, K. Lee ${ }^{c}$, and N. J. Ekins-Daukes ${ }^{c}$ \\ ${ }^{a}$ Department of Physics, Lancaster University, Lancaster, LA1 4YB, UK \\ b Department of Physics, Nelson Mandela Metropolitan University, Port Elizabeth, South \\ Africa \\ c Blackett Laboratory, Imperial College London, Prince Consort Road, London SW7 2BZ, UK \\ ${ }^{\mathrm{d}}$ Asahi Kasei Corporation, 2-1 Samejima, Fuji-city, Shizuoka 416-8501, Japan \\ * Corresponding author, telephone: +44 01524 592780, e-mail: fujita.hcc@asahi-kasei.co.jp
}

\begin{abstract}
We report on the open circuit voltage recovery in GaSb quantum ring (QR) solar cells under high solar concentration up to 2500 suns. The detailed behaviour of type II GaSb/GaAs QR solar cells under solar concentration, using different temperatures and light illumination conditions is analyzed through optical and electrical measurements. Although enhancement of the short-circuit current was observed due to sub-bandgap photon absorption in the QR, the thermionic emission rate of holes was found to be insufficient for ideal operation. The direct excitation of electron-hole pairs into QRs has revealed that the accumulation of holes is one of the causes of the open-circuit voltage ( $\mathrm{V}_{\mathrm{OC}}$ ) degradation. However, using concentrated light up to 2500 suns, the GaSb QR cell showed much quicker $\mathrm{V}_{\mathrm{OC}}$ recovery rate than a GaAs control cell.
\end{abstract}

Key words

Solar cell

Quantum dots and rings

Gallium antimonide

Concentration 
There is increasing interest in embedding quantum dots (QDs) or quantum rings (QRs) into conventional single junction solar cells for efficiency enhancement [1]. This improvement is expected to be achieved by sub-bandgap photon absorption below the absorption edge of the host material. There have been a few reports on structures such as InAs/GaAs QDs [2-4], InGaAs/GaAs QDs [5], and $\mathrm{GaSb} / \mathrm{GaAs}$ QDs [6, 7], showing enhanced infrared absorption. However, the embedment of QD layers into $\mathrm{GaAs}$ solar cells results in significant degradation of the open circuit voltage $\left(\mathrm{V}_{\mathrm{OC}}\right)$. Type II GaSb/GaAs QRs are attractive because of the staggered band offset where holes are localized within the GaSb QRs, but there is no electron confinement. Compared to Type I InAs QDs, this produces a larger red-shift of the photo-response and will capture more of the infrared solar spectrum, increasing the short-circuit current [8]. The reduced electron-hole wave function overlap results in increased carrier lifetimes which should reduce recombination and improve the extraction of photogenerated carriers [9]. Furthermore, it is desirable that electrons escape prior to heavy holes to prevent weakening of the built-in electric field, which reduces the open-circuit voltage [10]. Since there is no electron confinement in type II GaSb QRs photo-generated electrons are directly excited to the GaAs where they are free to drift under the influence of the internal electric field. The incorporation of type-II GaSb QDs has also been proposed as a way to realize an intermediate band solar cell, because the spatial separation of electron and holes can be engineered provide an intermediate band closer to the theoretical optimum and reduces unwanted thermal emission and capture processes [11,12]. However, the requirements for intermediate band solar cells are different to those for quantum dot solar cells. In this respect the larger effective mass of the holes in GaSb compared with InAs could result in more confined states with a consequent reduction in the separation between the QD confined states and the valence band, which can ultimately result in the reduction of the effective band gap of the cell and a reduction in the Voc. Meanwhile, compared to GaSb QD the GaSb QR nanostructures, can provide epitaxial layers having less strain [13], which is very beneficial in stacking such layers to enhance the light absorption at longer wavelength. Recently, GaSb/GaAs QR solar cells with extended infrared response up to $1400 \mathrm{~nm}$ have been demonstrated [13]. Furthermore, in this system, it has also been shown that the sub-bandgap response is limited by the thermionic emission of holes from QRs [14]. However, in order to further improve the solar cell efficiency, a more detailed understanding of the photo-carrier dynamics is essential, especially under high solar concentration conditions under which these solar cells will be implemented.

In this paper, we report on the open-circuit voltage recovery in GaSb QR solar cells under high solar concentration up to 2500 suns. The solar cell characteristics and the detailed behaviour of the photo-generated carriers in relation to the $\mathrm{GaSb} \mathrm{QR}$ within the active region were further 
characterized by investigation of the optical and electrical properties at different temperatures and under different illumination conditions.

\section{Experiment}

The GaSb QR solar cells were fabricated on n+-GaAs (100) substrates by molecular beam epitaxy (MBE). Ten layers of nominally 2.1 ML thick GaSb QRs grown at $480{ }^{\circ} \mathrm{C}$ were inserted into the intrinsic region of an otherwise conventional GaAs p-i-n structure. The growth of each QR layer was followed by a $5 \mathrm{~nm} \mathrm{GaAs}$ 'cold cap' layer grown at $430{ }^{\circ} \mathrm{C}$ and a $30 \mathrm{~nm}$ GaAs spacer layer grown at $570{ }^{\circ} \mathrm{C}$. The QR layers obtained above had an areal density of $\sim 1 \times 10^{10} \mathrm{~cm}^{-2}$ and were $1.7 \mathrm{~nm}$ in height, with a $23 \mathrm{~nm}$ outer diameter and a $10 \mathrm{~nm}$ inner diameter. The details of the growth process and structure have been described previously elsewhere [13]. The above structure was processed into circular solar cells having either a $1 \mathrm{~mm}$ or a $3.5 \mathrm{~mm}$ diameter active area, with $\mathrm{Au} / \mathrm{Zn} / \mathrm{Au}$ top and $\mathrm{Au} / \mathrm{InGe}$ bottom electrodes and an anti-reflection coating. Temperature dependent dark and light current density (J) - voltage (V) curves were obtained using a liquid nitrogen cooled cryostat. Illumination was provided by a calibrated tungsten-halogen light source where the maximum achievable concentration was equivalent to 19 suns. In order to clarify the behaviour of the photo-generated carriers in the GaSb QRs, a $1064 \mathrm{~nm}$ infrared laser was used to illuminate the device during the $\mathrm{J}-\mathrm{V}$ measurement at room temperature [14]. The wavelength is chosen so that it has small enough photon energy not to excite GaAs, but has large enough energy to excite electron-hole pairs in the GaSb QRs. The external quantum efficiency (EQE) was measured using a $0.3 \mathrm{~m}$ Bentham monochromator with Si and InGaAs detectors for incident light calibration. For high concentration measurements, a Xenon flash lamp was used as the light source which gave an illumination of around 2 milliseconds duration and the distance between the lamp and the solar cell was changed to vary the effective solar concentration ratio. The J-V measurements were carried out within $200 \mu \mathrm{sec}$ to prevent device heating, during which fluctuation of the illumination intensity was less than $\pm 5 \%$. The concentration ratio was calculated using the ratio of the short circuit current of a GaAs monitoring cell between 1 sun and the chosen concentration level, assuming that the shortcircuit current is proportional to the illumination intensity. The temperature and optical concentration dependences were measured using the $3.5 \mathrm{~mm}$ diameter solar cells with anti-reflection coating, whilst the laser illumination measurement was performed using the $1 \mathrm{~mm}$ diameter solar cells without antireflection coating

\section{Results and Discussion}

Figure 1 shows the J-V curves under 1 sun illumination and in the dark. The short-circuit current density (Jsc) is enhanced by $5.9 \%$ in the QR cell due to the additional absorption from sub-bandgap 
photon absorption [10]. However, a reduced open-circuit voltage (Voc) was observed, which is caused by the increased recombination current via the QRs and is evident from the increase in dark current as shown in the inset. Figure 2 shows a summary of the temperature dependence of the representative solar cell characteristics under 19 suns; i.e. short-circuit current density ( $\left.\mathrm{J}_{\mathrm{SC}}\right)$, opencircuit voltage $\left(\mathrm{V}_{\mathrm{OC}}\right)$, fill factor $(\mathrm{FF})$ and efficiency. Both the QR sample and the control sample show a constant increase of $\mathrm{J}_{\mathrm{SC}}$ with increasing temperature, resulting from the increased photo-absorption associated with the GaAs bandgap narrowing. At low temperature (100 K), $\mathrm{J}_{\mathrm{SC}}$ for the GaSb QR cell is lower than that of the GaAs control. However, $\mathrm{J}_{\mathrm{SC}}$ starts to increase rapidly above $180 \mathrm{~K}$, and surpasses the GaAs control cell above $250 \mathrm{~K}$. This behaviour is consistent with the observation that the photocurrent due to sub-bandgap photon absorption is dominated by thermionic emission of holes [14]. At the same time, Voc for the GaSb QR cell shows a slight change of slope, and the FF starts to decrease rapidly above $180 \mathrm{~K}$, resulting in a net reduction of the efficiency

The EQE spectra for the GaAs control and GaSb QR solar cells measured at $100 \mathrm{~K}$ and $290 \mathrm{~K}$ are shown in figure 3. Characteristics associated with the carrier behaviour in the GaSb QR are observed in the EQE spectra at low temperature. At room temperature $(290 \mathrm{~K})$, the EQE for both samples shows no significant difference except for the extended infrared response of the GaSb QR cell. However, at $100 \mathrm{~K}$ the EQE of the GaSb QR cell is notably reduced, especially at longer wavelengths (above $600 \mathrm{~nm}$ ). Because of the wavelength dependence of the absorption coefficient in GaAs, photons with short wavelength $(<500 \mathrm{~nm})$ are mainly absorbed within the emitter of the solar cell $(<0.5 \mu \mathrm{m})$, whilst photons with long wavelengths $(>700 \mathrm{~nm})$ can easily penetrate a few microns of the GaAs. For short wavelengths, photo-excited minority carriers (electrons) in the top p-GaAs region diffuse towards the n-GaAs bottom region via the intrinsic region. There is no electron confining potential around the $\mathrm{QR}$, therefore, electrons can easily pass through the intrinsic region. On the other hand, for long wavelengths, photo-excited minority carriers (holes) generated within the $\mathrm{n}-\mathrm{GaAs}$ base of the cell, diffuse towards the p-GaAs top via the intrinsic region. Due to the large valence band offset at the GaAs/GaSb QR interface, some of these holes become trapped by the QRs and must be extracted by thermal excitation in order to contribute to the external photocurrent. This thermal extraction of holes is significantly reduced at low temperatures, leading to the observed decrease in the EQE. The small reduction in room temperature EQE at long wavelengths (around $800 \mathrm{~nm}$ ) suggests that the rate of thermionic hole emission might be insufficient at room temperature.

Figure 4 shows the J-V characteristics for different light illumination conditions under a bias light of 4 suns (where the solid curve is measured under the bias light as a reference). The weaker white light and the $1064 \mathrm{~nm}$ infrared laser are superposed on top of the bias light and the results are shown as the dotted and dashed J-V curves, respectively. A schematic band-diagram of a GaSb QR under infrared 
laser illumination is shown in the inset. The intensity of the additional light source was adjusted to give the same short-circuit current in each case. Superposing the white light slightly increases the open-circuit voltage, as expected from the $\mathrm{V}_{\mathrm{OC}}-\mathrm{J}_{\mathrm{SC}}$ relation. On the other hand, the open-circuit voltage drops by $5.2 \%$ with additional infrared laser illumination. This behaviour can be understood by the increase in recombination current due to the accumulation of holes in the QRs. The infrared laser directly excites electron-hole pairs in GaSb QRs. As mentioned above, although electrons can easily drift into the n-region, holes remain trapped in the QRs. This increase in the hole accumulation makes QRs more attractive to electrons, leading to an increased recombination current. In this sense, rapid hole extraction from the QRs is essential, not only for increasing the photocurrent, but also for increasing (not degrading) the open-circuit voltage. The illuminated laser intensity (2.6 $\mathrm{Wcm}^{-2}$ ) corresponds to a concentration of roughly 200 suns. This means the concentrated operation of GaSb QR solar cells could further degrade $\mathrm{V}_{\mathrm{OC}}$ due to hole accumulation, unless thermal extraction can be increased.

Figure 5 shows the optical concentration dependence of $\mathrm{V}_{\mathrm{OC}}$ for the GaAs control and GaSb QR cells. The dashed, dotted and dashed-dotted lines are guides to the eye showing slopes of constant ideality factor (n), and the solid lines are the estimated $V_{O C}$ behaviour calculated from dark $\mathrm{J}-\mathrm{V}$ characteristics in the inset of figure 1. (These lines deviate at high concentration due to the effect of the series resistance). Although the GaSb QR cell shows a lower $V_{\text {OC }}$ than the GaAs control cell, a much quicker (+66 \%) open-circuit voltage recovery rate was observed for the GaSb QR solar cell (+98.3 mV/decade) compared to the GaAs control cell (+59.2 $\mathrm{mV} /$ decade) in the high concentration regime, reaching $\mathrm{V}_{\mathrm{OC}}=0.916 \mathrm{~V}$ at around 2500 suns. This voltage recovery rate is consistent with the equation below, which is derived from the conventional diode equation

$\Delta V_{O C} \cong \mathrm{nV}_{\mathrm{T}} \times \ln (\alpha)$

where $\mathrm{n}$ is the ideality factor, $\alpha$ is the concentration ratio and $\mathrm{V}_{\mathrm{T}}$ is the thermal voltage $\left(\mathrm{V}_{\mathrm{T}}=25.9 \mathrm{mV}\right.$ at $300 \mathrm{~K}$ ). The GaAs control cell shows high $\mathrm{V}_{\mathrm{OC}}$ recovery with large ideality factor $(\mathrm{n}=3.5)$ at low concentration, probably due to the surface recombination at the top and/or edge of the device. For a concentration ratio higher than 1 sun, $V_{\text {OC }}$ recovers according to the curve with $n=1$, which is consistent with a diffusion current dominated behaviour. On the other hand, the GaSb QR cell shows monotonic $\mathrm{V}_{\mathrm{OC}}$ recovery from the low to high concentration regime with $\mathrm{n}=1$.6. This means that $\mathrm{QR}$ related recombination does not saturate up to 2500 suns, indicating fast hole capture and recombination within the GaSb QRs. 


\section{Conclusions}

We investigated the optical and electrical behaviour of GaSb QR solar cells under concentration levels up to 2500 suns and characterized their temperature dependence, solar concentration dependence and infrared laser irradiation effects. A rapid increase of $\mathrm{J}_{\mathrm{SC}}$ was observed above $180 \mathrm{~K}$ due to the increase of thermionic extraction of holes from the GaSb QRs, and $\mathrm{J}_{\mathrm{SC}}$ enhancement reached $+5.9 \%$ compared to the GaAs control cell at room temperature (under 1 sun). However, degradation of $\mathrm{V}_{\mathrm{OC}}$ and $\mathrm{FF}$ was observed on approaching room temperature, leading to a net efficiency reduction. Using the direct excitation technique, hole accumulation in the QRs was found to be responsible for the $\mathrm{V}_{\mathrm{OC}}$ degradation. Concentration dependent measurements showed a $66 \%$ quicker $\mathrm{V}_{\text {OC }}$ recovery rate in the GaSb QR solar cell (98 mV/decade) and $\mathrm{V}_{\mathrm{OC}}$ reached $0.916 \mathrm{~V}$ at 2500 suns. In order to further improve the solar cell performance, hole capture by QRs must be reduced, and/or the hole extraction rate from the QRs must be increased, not only for photocurrent enhancement but also to improve $\mathrm{V}_{\mathrm{OC}}$. Nevertheless, the increase in $\mathrm{V}_{\mathrm{OC}}$ under concentration of our QR solar cell is an encouraging sign.

Acknowledgements

This work was supported by the EPSRC (grants EP/E028209/1 and EP/G070334/1) and the EU Marie-Curie Training networks (PROPHET) PITN-GA-2010-264687 and (PROMIS) H2020-MSCAITN-2014-641899. H. Fujita would like to thank Asahi Kasei Corporation for the award of an industry scholarship. P. J. Carrington and A.R.J. Marshall gratefully acknowledge support as fellowships from the Royal Academy of Engineering. 


\section{References}

[1] A. Luque and A. Marti, Phys. Rev. Lett. 78, 265014 (1997).

[2] S. M. Hubbard, C. D. Cress, C. G. Bailey, R. P. Rafaell, S. G. Bailey, and D. M. Wilt, Appl. Phys. Lett. 92, 123512 (2008).

[3] A. Luque, A. Marti, N. Lopez, E. Antolin, and E. Canovas, J. Appl. Phys. 99, 094503 (2006).

[4] R. Oshima, A. Takata, and Y. Okada, Appl. Phys. Lett. 93, 083111 (2008).

[5] T. Sugaya, S. Furue, H. Komaki, T. Amano, M. Mori, K. Komori, S. Niki, O. Numakami, and Y. Okano, Appl. Phys. Lett. 97, 183104 (2010).

[6] R. B. Laghumavarapu, A. Moscho, A. Khoshakhlagh, M. El-Emawy, L. F. Lester, and D. L. Huffaker, Appl. Phys. Lett. 90, 173125 (2007).

[7] R. B. Laghumavarapu, B. L. Liang, Z. S. Bittner, T. S. Navruz, S. M. Hubbard, A. Norman, and D. L Huffaker, Solar Energy Materials and Solar Cells 114, 165 (2013).

[8] P.J. Carrington, A.S. Mahajumi, M.C. Wagener, J.R. Botha, Q. Zhuang and A. Krier, Physica B 407, 1493 (2012)

[9] J. Hwang, A. J. Martin, J. M. Millunchick, and J. D. Phillips, J. Appl. Phys. 111, 074514 (2012).

[10] A. Alemu, J. A. H. Coaquira, and A. Freundlich, J. Appl. Phys. 99, 084506 (2006).

[11] J. Wu, D. Shao, Z. Li, M. O. Manasreh, V.P. Kunets, Z.M.Wang and G. J. Salamo, Appl. Phys. Lett. 95, 071908 (2009)

[12] P. G. Linares, A. Martí, E. Antolín, C.D. Farmer, Í. Ramiro, C.R. Stanley and A. Luque, Sol. Energy Mat. Sol. Cells 98, 240 (2012)

[13] P. J. Carrington, M. C. Wagener, J. R. Botha, A. M. Sanchez, and A. Krier, Appl. Phys. Lett. 101, 231101 (2012).

[14] H. Fujita, J. James, P. J. Carrington, A. R. J. Marshall, A. Krier, M. C. Wagener, and J. R. Botha, Semicond. Sci. Technol. 29, 035014 (2014). 